\title{
NMR of Insensitive Nuclei Enhanced by Dynamic Nuclear Polarization
}

\author{
Pascal Miéville§, Sami Jannin*, Lothar Helm, and Geoffrey Bodenhausen \\ $\S S C S$ DSM Prize for best poster
}

\begin{abstract}
Despite the powerful spectroscopic information it provides, Nuclear Magnetic Resonance (NMR) spectroscopy suffers from a lack of sensitivity, especially when dealing with nuclei other than protons. Even though NMR can be applied in a straightforward manner when dealing with abundant protons of organic molecules, it is very challenging to address biomolecules in low concentration and/or many other nuclei of the periodic table that do not provide as intense signals as protons. Dynamic Nuclear Polarization (DNP) is an important technique that provides a way to dramatically increase signal intensities in NMR. It consists in transferring the very high electron spin polarization of paramagnetic centers (usually at low temperature) to the surrounding nuclear spins with appropriate microwave irradiation. DNP can lead to an enhancement of the nuclear spin polarization by up to four orders of magnitude. We present in this article some basic concepts of DNP, describe the DNP apparatus at EPFL, and illustrate the interest of the technique for chemical applications by reporting recent measurements of the kinetics of complexation of ${ }^{89} \mathrm{Y}$ by the DOTAM ligand.
\end{abstract}

Keywords: DNP $\cdot$ Contrast agent $\cdot$ Kinetics $\cdot$ NMR $\cdot$ Yttrium

\section{Polarization in Magnetic Resonance Experiments}

Magnetic Resonance (MR) spectroscopy consists of exciting and measuring transitions between spin states. At thermal equilibrium, the population of each spin state is determined by the Boltzmann distribution. The difference of populations (normalized to the total number of spins) defines the spin polarization. For a spin $I=1 / 2$ of gyromagnetic ratio $\gamma_{\mathrm{I}}$ placed in a magnetic field $B_{0}$ at a temperature $T$, two spin states $\alpha$ and $\beta$ exist and the spin polarization $P$ is

$$
P=\frac{\Delta N}{N}=\tanh \left(\frac{\gamma h B_{0}}{2 k_{B} T}\right)
$$

where $N$ is the total number of spins, $\Delta N$ the difference of spin populations between the two states $\alpha$ and $\beta, h$ the Planck constant, and $k_{\mathrm{B}}$ the Boltzmann constant. As

${ }^{\star}$ Correspondence: P. Miéville; Dr. S. Jannin Ecole Polytechnique Fédérale de Lausanne Institut des Sciences et Ingénierie Chimiques Batochime

$\mathrm{CH}-1015$ Lausanne

Tel.: +41216939724

Fax: + 41216939435

E-mail: pascal.mieville@epfl.ch the MR signal intensity $s(t)$ is directly related to the polarization (Eqn. (2)), increasing the polarization increases the MR signal intensity after excitation, hence the sensitivity.

$$
s(t) \propto P N
$$

\section{Principle of Dynamic Nuclear Polarization (DNP)}

Eqn. (1) indicates that the spin polarization can be significantly increased by lowering the temperature or increasing the magnetic field, hence the Larmor frequency which is proportional to $\gamma_{\mathrm{I}}$ and $B_{0}$. Under conventional ${ }^{1} \mathrm{H}$ Nuclear Magnetic Resonance conditions (at thermal equilibrium with $T=298 \mathrm{~K}$ and $B_{0}=3.35$ Tesla), the proton spin polarization is $P_{\mathrm{I}}=1.2 \times$ $10^{-5}$. Due to its higher gyromagnetic ratio $\gamma_{\mathrm{e}}$ the spin polarization of an electron spin $S=1 / 2$ is $\sim 660$ times larger: $P_{\mathrm{e}}=7.2 \times 10^{-3}$. Furthermore, if the temperature is lowered down to $\sim 1 \mathrm{~K}$, the electron spin polarization gets close to its theoretical maximum $\left(P_{\mathrm{e}} \sim 1\right)$. The maximum nuclear spin polarization however is still only $1.5 \times 10^{-3}$ at $1 \mathrm{~K}$ (Fig. 1).

The global idea of DNP consists in the transfer of the nearly complete electron spin polarization at very low temperature to the nuclear spins of a molecule of interest in order to increase substantially the NMR sensitivity of the latter. A typical sample for a Dynamic Nuclear Polarization experiment must therefore be a frozen

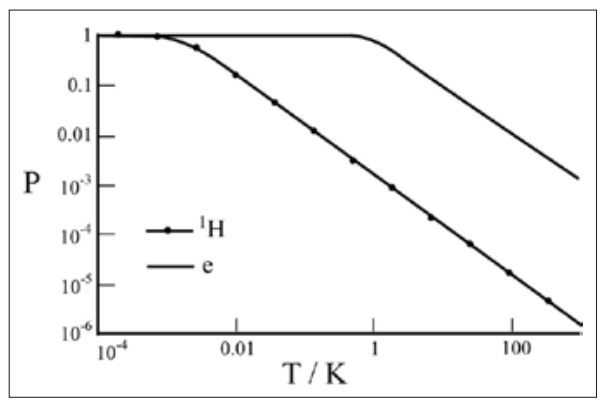

Fig. 1. Polarization of the $1 / 2$ spins of the electron (plain line) and of the ${ }^{1} \mathrm{H}$ nucleus (line with dots) as a function of the temperature in Kelvin at 3.35 T.

mixture of the compound to be studied together with paramagnetic centers. The mixture of the paramagnetic centers (such as organic radicals) with the molecules of interest in a glass-forming solvent (typically water/glycerol or water/ethanol), prepared at room temperature, is rapidly frozen and placed into a DNP polarizer ${ }^{[1]}$ with a moderate magnetic field $\left(B_{0}=3.35\right.$ $\mathrm{T}$, corresponding to an electron spin Larmor frequency of about $f=94 \mathrm{GHz}$ ) at low temperature $(T \sim 1.2 \mathrm{~K})$. A glassy matrix, in contrast to a crystalline one, is important to ensure a homogenous medium where the polarization can diffuse efficiently. The high electron spin polarization is transferred to the surrounding nuclear spins by a microwave irradiation applied at an optimum frequency, for example $93.89 \mathrm{GHz}$ for the free radical TEMPO and optimum power of typically $P_{\text {uw }}=30 \mathrm{~mW}$. A net increase of nuclear spin polarization is cre- 
ated close to the paramagnetic centers and diffuses rapidly through the entire sample. The polarization diffusion gives rise to a sample where the nuclear spins are highly and 'homogeneously' polarized. Typically nuclear spin polarizations of $P_{\mathrm{I}}=40 \%$ for ${ }^{1} \mathrm{H}$ and $P_{\mathrm{I}}=10 \%$ for ${ }^{13} \mathrm{C}$ spins are obtained. Depending on the electron spin resonance (ESR) properties of the paramagnetic radical used as a source of polarization (ESR lineshape, $T_{1}, T_{2}$, etc.) as well as on the temperature $T$ and magnetic field $B_{0}$, DNP can occur through two different mechanisms: the Solid Effect (SE) and Thermal Mixing (TM). The theoretical descriptions of these mechanisms are rather complex and have been thoroughly described in the literature by Abragam and Goldman (see ref. [2] for an exhaustive review on the subject). The SE can occur when the ESR lines are narrow, i.e. when the root of the second moment of the ESR spectrum, $M_{2}{ }^{1 / 2}$, is smaller than the NMR resonance frequency $f_{\mathrm{I}}$, which is typical for a radical concentration $<10^{18} \mathrm{spins} \cdot \mathrm{cm}^{-3}$. To achieve the SE, the ESR line is irradiated at an offset $\pm \omega_{n}$ from the center of the spectrum $\omega_{e}$. Such an irradiation excites a second-order transition (also called electron-nucleus flip-flop or 'forbidden' transition), which leads to a straightforward increase in nuclear spin polarization (see Fig. 2).

For higher radical concentrations, the ESR resonance becomes broader $\left(M_{2}^{1 / 2}>\right.$ $f_{\mathrm{I}}$ ) and the Thermal Mixing (TM) ${ }^{[3]}$ mechanism becomes dominant. TM usually requires less microwave power than $\mathrm{SE}$, typically one to two orders of magnitude. It is also advantageous in the sense that it results in an optimum polarization for all nuclear spins present in the sample at a unique microwave frequency (in contrast to SE where an optimum microwave frequency has to be found for each nuclear spin $\gamma_{\mathrm{I}}$ ). TM involves a large number of coupled electron spins and its description is much more complex than that of SE. The ensembles of electron and nuclear spins behave like energy reservoirs so that a thermodynamic model turns out to be more appropriate than a quantum mechanical description. ${ }^{[4]}$ Following such a model, the effect of microwaves is to 'cool down' the electron spin dipolar reservoir to a temperature $T_{\mathrm{s}}$. Subsequently the nuclear spin thermal reservoir tends to equalize its temperature to $T_{\mathrm{S}}$ by 'thermal contact'. The final polarization is given by Eqn. (1) where the sample temperature $T$ is replaced by $T_{\mathrm{S}}$. The basics of TM were addressed by Goldman in a recent publication. ${ }^{[5]}$ The experimental conditions described $(T \sim 1.2 \mathrm{~K}$ and $\left.B_{0}=3.35 \mathrm{~T}\right)$ and the ESR properties of the paramagnetic molecule used for DNP (in our case the free radical TEMPO at a concentration of $30 \mathrm{mM}$, with $M_{2}^{1 / 2}>f_{\mathrm{I}}$ ) give rise to TM as dominant mechanism. The

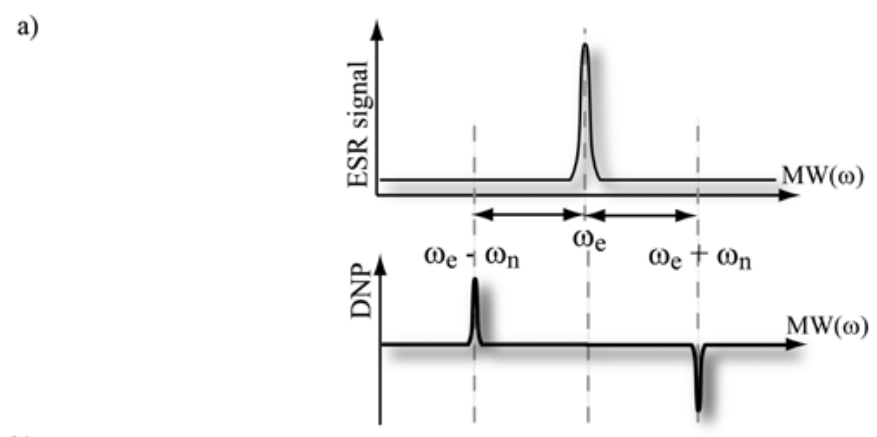

b)

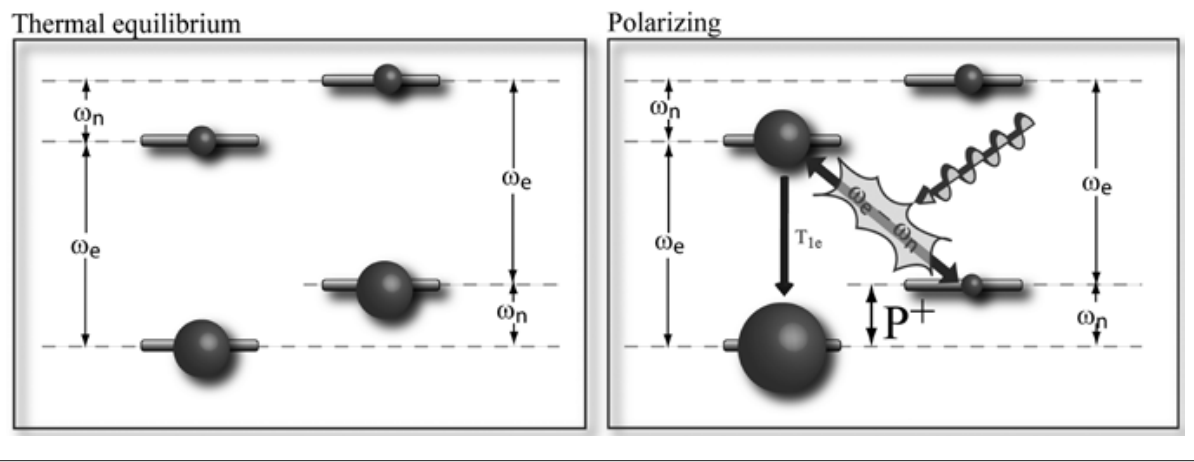

Fig. 2. a) ESR line and DNP transitions for a microwave frequency $\omega_{\mu w}=\omega_{e} \pm \omega_{n}$ b) Solid Effect DNP scheme for a simple spin system consisting of a single nuclear spin I = $1 / 2$ and a single electron spin $S=1 / 2$ (the sizes of the grey spheres represent populations).

resulting polarization observed on several nuclear spins follow the rule $P_{\mathrm{I}} \propto \gamma_{\mathrm{I}}$ (if $P_{1 \mathrm{H}}$ $=40 \%, P_{13 \mathrm{C}}=10 \%$, etc. .)

\section{DNP Hardware and Technique}

DNP is performed at EPFL in a 'homebuilt' polarizer. ${ }^{[1,6,7]}$ The apparatus consists of a continuous flow ${ }^{4} \mathrm{He}$ cryostat operating at $T=1.2 \mathrm{~K}$ in an Oxford Instruments wide bore cryomagnet with $B_{0}=3.35 \mathrm{~T}$. The cryostat is equipped with a home-built DNP probe (microwave irradiation at $f_{\text {uw }}=$ $93.5-94.5 \mathrm{GHz}$ and NMR detection at $f_{\mathrm{I}}=$ $10-150 \mathrm{MHz})$. The DNP probe is coupled to an ELVA microwave source providing a maximum power of $\mathrm{P}_{\mu \mathrm{w}}=200 \mathrm{~mW}$ and to an NMR spectrometer for monitoring the nuclear spin polarization as a function of time of microwave irradiation. The apparatus was developed at EPFL (LPMN) in close collaboration with the Paul Scherrer Institut (SEPT). The DNP samples are frozen to a glassy state, in liquid $\mathrm{N}_{2}$, prior to insertion into the polarizer. Visual inspection of the frozen matrix usually gives an indication that the sample is a homogeneous glass, ensuring that all molecules and radicals are randomly distributed and oriented, which is mandatory for efficient DNP. A good glass-forming mixture is generally made of $\sim 50 \% \mathrm{D}_{2} \mathrm{O}$ and $\sim 50 \%$ of a deuterated glass-forming solvent such as glycerol-d8, ${ }^{[8]}$ methanol-d4 or DMSOd6. The radicals TEMPO or TEMPOL are mixed with the solvent at a concentration of 30 to $50 \mathrm{mM}$ together with the target molecule(s). The solution is rapidly frozen to small beads of $c a$. 10-30 $\mu \mathrm{L}$ by dropping it into a liquid nitrogen bath. The beads are subsequently loaded into the sample holder (Fig. 3), placed inside the

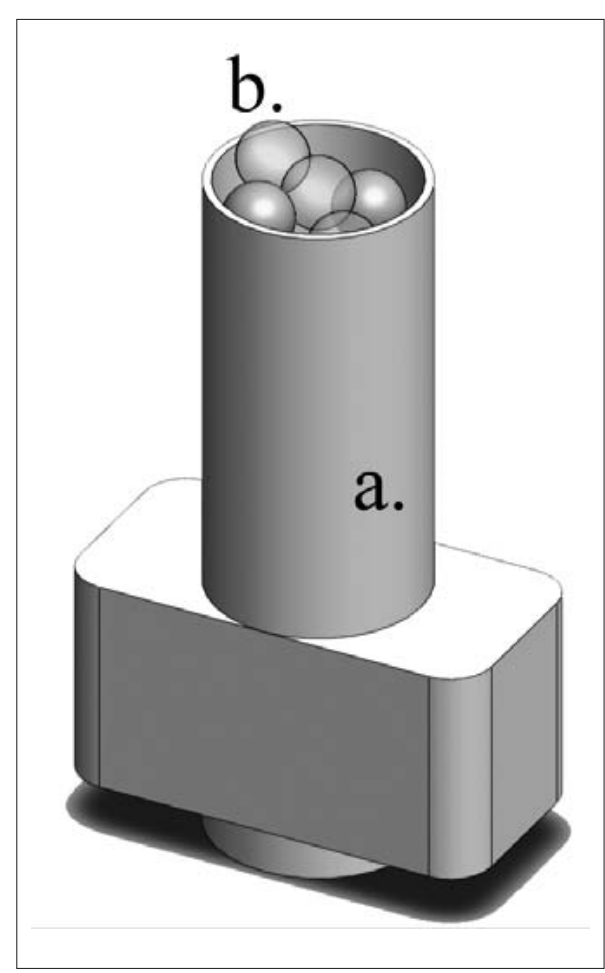

Fig. 3. a) $300 \mu \mathrm{L}$ PTFE sample holder; b) 10-30 $\mu L$ frozen sample beads. 
polarizer $\left(T=1.2 \mathrm{~K}\right.$ and $\left.B_{0}=3.35 \mathrm{~T}\right)$, and irradiated with $\sim 30 \mathrm{~mW}$ of microwaves power at $93.89 \mathrm{GHz}$. The build-up of the nuclear spin polarization can be observed by 'small angle' pulsed NMR.

\section{NMR Measurement of the Hyperpolarized Sample}

Apart from the measurement of the nuclear polarization that has been built-up in the solid state at $T=1.2 \mathrm{~K}$, NMR does not provide sufficient resolution under DNP conditions due to the large dipolar couplings on the order of tens of $\mathrm{kHz}$. The subsequent broadening masks chemical shift and scalar coupling information that is relevant for chemical structure determination. Therefore, the sample is rapidly brought back to the liquid state for normal high-resolution NMR measurements. This is performed by dissolving parts of the frozen solution with a hot burst of deuterated water vapor $(T=400 \mathrm{~K}$ and $P=1 \mathrm{MPa})$ while preserving the high spin polarization. The produced liquid is transferred to a conventional NMR spectrometer at a few meters from the polarizer through a $2 \mathrm{~mm}$ PTFE capillary using helium gas under an overpressure of $0.2-0.6 \mathrm{MPa}$ (Fig. 4). The polarization of the nuclear spins decreases exponentially with the longitudinal relaxation time $\mathrm{T}_{1}$ as time constant.

The overall procedure including DNP at low temperature and dissolution of the highly polarized sample (also called 'hyperpolarized sample') is called 'dissolution DNP'. ${ }^{[9,10]}$ Since its invention in 2005, it has found application in numerous NMR experiments where sensitivity is an issue that dissolution-DNP can overcome.

\section{DNP Enhanced NMR of Low- sensitivity Nuclei}

The gain in sensitivity induced by the DNP process allows the investigation of a class of low sensitivity nuclei that are poorly accessible to 'standard' NMR. Nuclei with a low $\gamma$ and long $\mathrm{T}_{1}$ (normally $\mathrm{S}=$ $1 / 2$ ) are difficult to observe as they require the acquisition of numerous scans $(>1000)$ and with a long relaxation delay ( $>100 \mathrm{~s})$ between scans. The gain of several orders of magnitude in sensitivity obtained by DNP allows the acquisition of NMR spectra with only one scan. After an NMR experiment using an optimal excitation pulse of $90^{\circ}$, all the hyperpolarization is lost. The pulse angle used for NMR excitation can however be smaller than $90^{\circ}$, preserving partially the high nuclear spin polarization. In that way the NMR signal can be recorded more than once, thus allowing for example relaxation time measurements. The

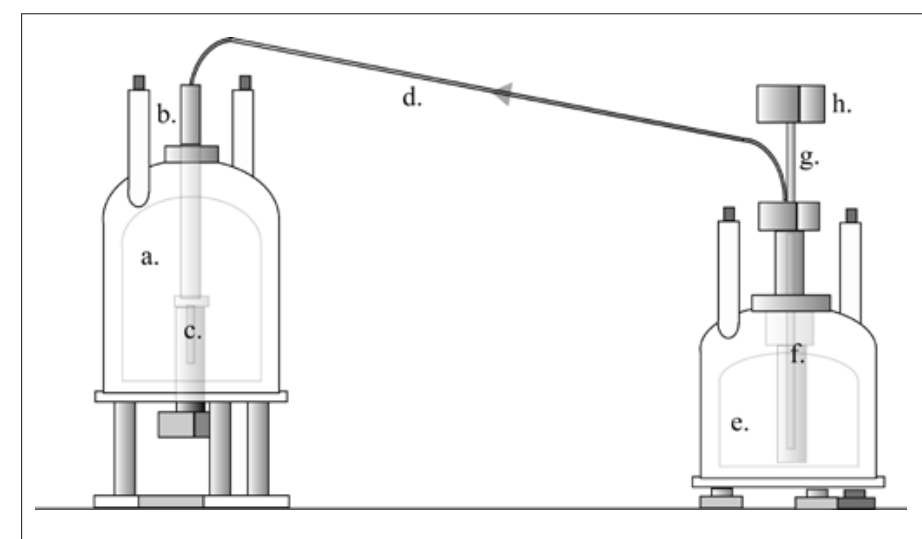

Fig. 4. Dissolution DNP setup, a) NMR cryomagnet $(7.05$ $\mathrm{T)}$; b) polarized solution injector; c) NMR tube sitting in the NMR probe; $d$ ) PTFE transfer line; e) DNP cryomagnet (3.35 T); f) cryostat; g) waveguide; h) microwave source.

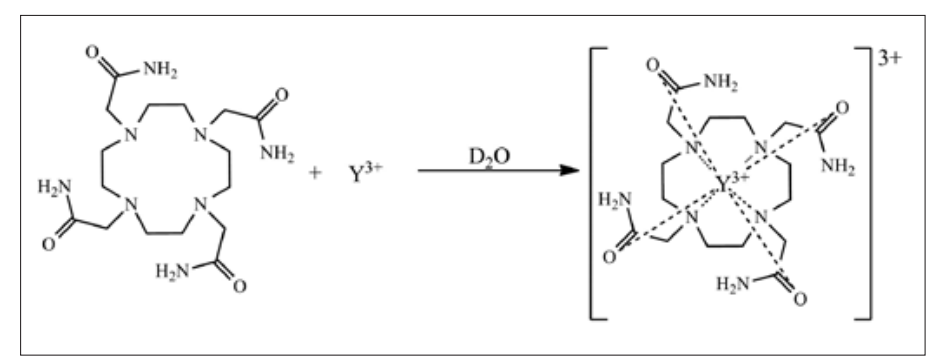

Scheme 1. Scheme of the complexation reaction between yttrium and DOTAM.

very long $\mathrm{T}_{1}$ of the low $\gamma$ nuclei becomes an advantage as it preserves the polarization for a long time and allows to follow the dynamics of chemical processes such as complexation. Some potentially interesting $\mathrm{S}=1 / 2$ nuclei are ${ }^{13} \mathrm{C},{ }^{15} \mathrm{~N},{ }^{77} \mathrm{Se}$ (relative sensitivity to $\left.{ }^{1} \mathrm{H} \mathrm{D}^{\mathrm{p}}: 5.37 \times 10^{-4}, \mathrm{~T}_{1}: \sim 30 \mathrm{~s}\right)$, ${ }^{57} \mathrm{Fe}\left(\mathrm{D}^{\mathrm{p}}: 7.24 \times 10^{-7}, \mathrm{~T}_{1}: \sim 3 \mathrm{~s}\right)$ or ${ }^{107} \mathrm{Ag}\left(\mathrm{D}^{\mathrm{p}}\right.$ : $\left.3.50 \times 10^{-5}, \mathrm{~T}_{1}: \sim 400 \mathrm{~s}\right)$.

\section{Examples of Applications}

Many experiments have been performed showing the interest of DNP, such as ${ }^{129} \mathrm{Xe}$ hyperpolarization, ${ }^{[11]}$ tumor cell detection via hyperpolarized ${ }^{13} \mathrm{C}$ pyruvate, ${ }^{[12]}$ or surface NMR in porous solids (MAS-DNP). [13] We demonstrate an application of DNP to the very insensitive yttrium-89 nucleus. DNP-enhanced yttrium-89 (spin 1/2, $\left.\mathrm{T}_{1} \sim 600 \mathrm{~s}\right)^{[14]}$ is a very good model for paramagnetic gadolinium (same $3^{+}$charge, similar ionic radius, same coordination number) used as contrast agent for Magnetic Resonance Imaging (MRI). ${ }^{[15]}$ Hyperpolarized ${ }^{89} \mathrm{Y}$ is a sensitive NMR probe and allows the kinetics of the formation of the complex $\left[\mathrm{Y}(\mathrm{DOTAM})\left(\mathrm{D}_{2} \mathrm{O}\right)\right] \mathrm{Cl}_{3}$ to be followed 'on the fly' by dissolution-DNP. The experiment described here consisted in polarizing a frozen solution of $1 \mathrm{M} \mathrm{YCl}_{3}$ in D O/glycerol-d6 (40/60 w\%) doped with $30 \mathrm{mM}$ of TEMPOL free radical at 1.2 $\mathrm{K}$ and $3.35 \mathrm{~T}$ with microwave irradiation at $f_{\mu \mathrm{w}}=93.89 \mathrm{GHz}\left(P_{\mu \mathrm{w}}=30 \mathrm{~mW}\right)$. The polarized sample was rapidly dissolved with $5 \mathrm{~mL}$ of $\mathrm{D}_{2} \mathrm{O}$ pre-heated to $T=400$ $\mathrm{K}$ at a pressure $P=1 \mathrm{MPa}$ and transferred to a 7.05 $\mathrm{T}$ Bruker NMR magnet (300
$\mathrm{MHz}$ for protons). A $5 \mathrm{~mm}$ NMR sample tube containing $250 \mu \mathrm{L}$ of a DOTAM (1,4,7,10-tetrakis(acetamido)-1,4,7,10-tetraazacyclododecane) solution at $296 \pm 0.5$ $\mathrm{K}$, buffered with $1 \mathrm{M}$ of urotropine $(\mathrm{pH}=$ 5.01 with the ionic strength stabilized with $1 \mathrm{M} \mathrm{KCl}$ ) is placed in the NMR spectrometer, locked and shimmed. $250 \mu \mathrm{L}$ of the hyperpolarized ${ }^{89} \mathrm{Y}$ solution were rapidly injected and mixed with the DOTAM solution and the NMR acquisition was started after $5 \mathrm{~s}$ to monitor the complexation reaction (Scheme 1).

The NMR acquisition consisted in a series of small RF pulses $\left(15^{\circ}\right)$ applied at 60 $\mathrm{s}$ intervals. Each of the ${ }^{89} \mathrm{Y}-\mathrm{NMR}$ spectra shows two resonance peaks corresponding to the free yttrium at $\delta=0 \mathrm{ppm}$ and to the complexed yttrium $\left[\mathrm{Y}(\mathrm{DOTAM})\left(\mathrm{D}_{2} \mathrm{O}\right)\right]^{3+}$ at $\delta=123$ ppm (Fig. 5a).

The time evolution of the NMR signal integral of each peak was fitted (Fig. 5b) with the following equations. The free yttrium signal is determined by the kinetics of complexation (decreasing concentration) and by its spin-lattice relaxation time $T_{1 Y^{\circ}}$ (Eqn. (3)) where $S_{Y}$ is the NMR signal of the free yttrium, $N$ is the number of RF pulses applied at intervals $\Delta t, \beta$ is the nutation angle, $[Y]$ and $[D]$ are respectively the yttrium and DOTAM concentrations, $k$ is the formation rate constant and $P_{\mathrm{Y} 0}$ is the initial ${ }^{89} \mathrm{Y}$ spin polarization at time $t=0$. The full derivation of Eqns. (3) and (4) is given in the supplementary information of ref. [15]. The NMR signal of the complexed form of yttrium depends on the complexation kinetics, and on the spin-lattice relaxation times $T_{1 \mathrm{Y}}$ and $T_{1 \mathrm{YD}}$ of free yttrium and of yttrium complexed with DOTAM: (Eqn. (4)) where $S_{Y D}$ is the NMR signal of 


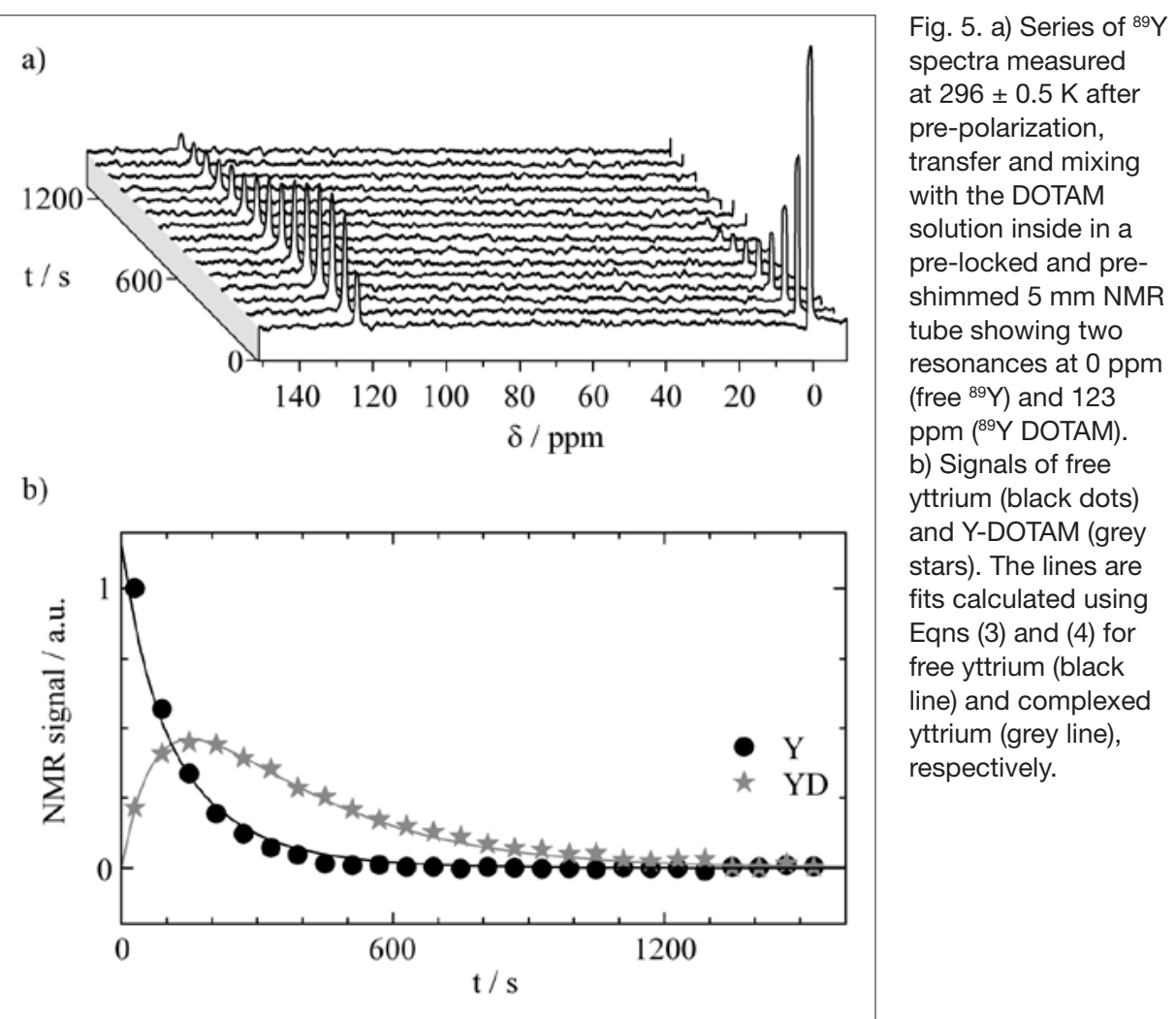

$S_{Y}(N \Delta t) \propto P_{Y 0} \sin (\beta) \cos ^{N}(\beta) e^{-\frac{N \Delta t}{T_{1 Y}}}\left([Y]_{0}-[D]_{0}[Y]_{0} \frac{e^{[Y]_{0} k N \Delta t}-e^{[D]_{0} k N \Delta t}}{[Y]_{0} e^{[Y]_{0} k N \Delta t}-[D]_{0} e^{[D]_{0} k N \Delta t}}\right)$

$S_{Y D}(N \Delta t) \propto P_{Y 0} \sin (\beta) \cos ^{N}(\beta) \int_{0}^{t}[Y D](\tau) e^{-\frac{\tau}{T_{1 Y}}} e^{-\frac{N \Delta t-\tau}{T_{1 X D}}} d \tau$

yttrium complexed with DOTAM and $[Y D]$ is the $\left[\mathrm{Y}(\mathrm{DOTAM})\left(\mathrm{D}_{2} \mathrm{O}\right)\right]^{3+}$ concentration. With $\mathrm{T}_{1 \mathrm{Y}}=1277 \mathrm{~s}$ and $\mathrm{T}_{1 \mathrm{YD}}=300 \mathrm{~s}$, the formation rate constant extracted from the fit gives $k=0.095 \mathrm{~s}^{-1} \mathrm{M}^{-1}$ which is close to the value of $\mathrm{k}=0.097 \mathrm{~s}^{-1} \mathrm{M}^{-1}$ determined by Baranyai et al. in 2007.[16] This examples demonstrates that it is possible to follow chemical reactions using hyperpolarized slowly relaxing nuclei provided the characteristic half-life $\tau=1 / k$ is short compared to the longitudinal relaxation times $T_{1}$.

\section{Acknowledgments}

We thank Dr. Jacques van der Klink and Dr. Arnaud Comment of EPFL and Dr. Ben van den Brandt, Dr. Jacobus Konter, and Dr. Patrick Hautle of the Paul Scherrer Institute for the design and construction of the DNP polarizer. We gratefully acknowledge Martial Rey for technical support. We thank Dr. Paul Vasos, Dr. Riddhiman Sarkar and Dr. Puneet Ahuja for their contributions. This work was supported by the Swiss National Science Foundation (FNS Grant 200020_124694 to G.B. and Paul Vasos), the Commission pour la Technologie et l'Innovation (CTI), the Ecole Polytechnique Fédérale de Lausanne (EPFL), and the French CNRS.

Received: January 24, 2011
Montet, F. Lazeyras, J. P. Vallee, P. Hautle, J. A. Konter, B. van den Brandt, J. P. Ansermet, R. Gruetter, G. Bodenhausen, Phys. Rev. Lett. 2010, 105.

[12] K. Golman, J. S. Petersson, Acad. Radiol. 2006 13, 932 .

[13] A. Lesage, M. Lelli, D. Gajan, M. A. Caporini, V. Vitzthum, P. Miéville, J. Alauzun, A. Roussey, C. Thieuleux, A. Mehdi, G. Bodenhausen, C. Coperet, L. Emsley, J. Am. Chem. Soc. 2010, 132,15459 .

[14] M. E. Merritt, C. Harrison, Z. Kovacs, P. Kshirsagar, C. R. Malloy, A. D. Sherry, J. Am Chem. Soc. 2007, 129, 12942.

[15] P. Miéville, S. Jannin, L. Helm, G. Bodenhausen, J. Am. Chem. Soc. 2010, 132, 5006.

[16] Z. Baranyai, I. Banyai, E. Brucher, R. Kiraly, E Terreno, Eur. J. Inorg. Chem. 2007, 3639.
[1] A. Comment, B. van den Brandt, K. Uffmann, F. Kurdzesau, S. Jannin, J. A. Konter, P. Hautle, W. T. H. Wenckebach, R. Gruetter, J. J. van der Klink, Concept. Magn. Reson. B 2007, 31B, 255.

[2] A. Abragam, M. Goldman, Rep. Prog. Phys. 1978, 41, 395.

[3] 'Spin Temperature and Nuclear Magnetic Resonance in Solids', Ed. M. Goldman, Oxford University Press: Oxford, 1970.

[4] W. T. Wenckebach, Nucl. Instrum. Meth. A $1995,356,1$.

[5] F. Kurdzesau, B. van den Brandt, A. Comment, P. Hautle, S. Jannin, J. J. van der Klink, J. A. Konter, J. Phys. D Appl. Phys. 2008, 41.

[6] A. Comment, J. Rentsch, F. Kurdzesau, S. Jannin, K. Uffmann, R. van Heeswijk, P. Hautle, J. A. Konter, B. van den Brandt, J. J. van der Klink, J. Magn. Reson. 2008, 194, 152.

[7] A. Comment, B. van den Brandt, K. Uffmann, F. Kurdzesau, S. Jannin, J. A. Konter, P. Hautle, W. T. Wenckebach, R. Gruetter, J. J. van der Klink, Appl. Magn. Reson. 2008, 34, 313.

[8] B. van den Brandt, P. Hautle, J. A. Konter, F. Kurdzesau, Appl. Magn. Reson. 2008, 34, 475.

[9] J. H. Ardenkjaer-Larsen, B. Fridlund, A. Gram, G. Hansson, L. Hansson, M. H. Lerche, R. Servin, M. Thaning, K. Golman, Proc. Natl. Acad. Sci. USA 2003, 100, 10158

[10] A. M. Leach, P. Miller, E. Telfeyan, D. B Whitt, General Electric: USA, 2009, Vol. US2009263325-A1.

[11] A. Comment, S. Jannin, J. N. Hyacinthe, P. Miéville, R. Sarkar, P. Ahuja, P. R. Vasos, X. 